\author{
Adel Aukhatov
}

\title{
Durchgriffs- und Existenzvernichtungshaftung im deutschen und russischen Sach- und Kollisionsrecht
}

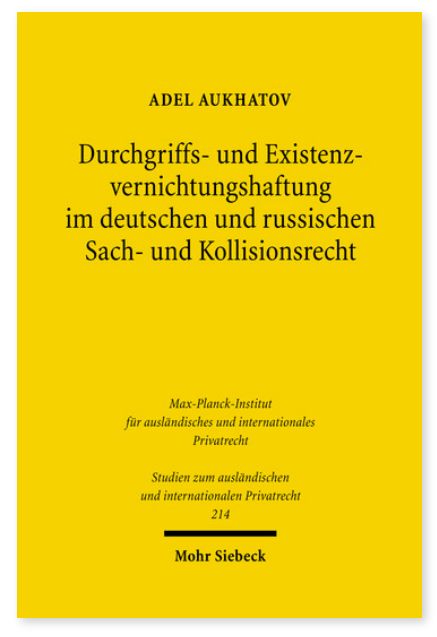

2009. XXI, 294 Seiten. StudIPR 214

ISBN 978-3-16-151399-2

DOI 10.1628/978-3-16-151399-2

eBook PDF 74,00€

ISBN 978-3-16-149885-5

fadengeheftete Broschur $74,00 €$
Adel Aukhatov analysiert vergleichend die Ausgangspunkte des Problems der Durchgriffs- und Existenzvernichtungshaftung im Sach- und Kollisionsrecht sowie Lösungsansätze in Deutschland und Russland. Für beide Rechtsordnungen gilt, dass die Durchgriffshaftung grundsätzlich nur subsidiär und als ultima ratio angewandt und in der Regel von den Umständen des Einzelfalls abhängig gemacht wird. Sie ist im deutschen wie im russischen IPR gesellschaftsrechtlich zu qualifizieren und damit einheitlich dem Gesellschaftsstatut zu unterstellen. Die kollisionsrechtlichen Instrumente (ordre public-Vorbehalt, Sonderanknüpfung und Gesetzesumgehung) helfen bei der Durchsetzung der russischen Kann-Bestimmungen über die Durchgriffshaftung der Gesellschafter in Russland agierender Auslandsgesellschaften nicht weiter. Anders ist die Situation in Deutschland. Die Durchgriffshaftung wegen Vermögensvermischung kann über Art. 6 EGBGB kraft der residualen positiven Funktion des ordre public oder als international zwingende Norm im Wege einer Sonderanknüpfung durchgesetzt werden. Die Existenzvernichtungshaftung ist nach dem »Trihotel«-Urteil des BGH eine auf § 826 BGB gestützte Innenhaftung der Gesellschafter gegenüber der Gesellschaft. Der Autor geht davon aus, dass sich ihre deliktsrechtliche Qualifikation durchsetzen wird, auch wenn die besseren Gründe für eine gesellschaftsrechtliche Qualifikation sprechen. Selbst auf der Basis einer deliktsrechtlichen Qualifikation der Existenzvernichtungshaftung ist aber deren akzessorische Anknüpfung an das Gesellschaftsstatut zu befürworten.

Adel Aukhatov Geboren 1978; Studium der Rechtswissenschaften in Kazan (Russische Föderation), Gießen, Köln und Kiel (LL.M.); 2005 Promotion an der Staatlichen V.I.Uljanov-Lenin-Universität zu Kazan; 2008 Promotion an der Rechtswissenschaftlichen Fakultät der Christian-Albrechts-Universität zu Kiel.
Jetzt bestellen:

https://mohrsiebeck.com/buch/durchgriffs-und-existenzvernichtungshaftung-im-deutschen-und-russischen-sach-undkollisionsrecht-9783161513992?no_cache=1

order@mohrsiebeck.com

Telefon: +49 (0)7071-923-17

Telefax: $+49(0) 7071-51104$ 